\title{
Inducing critical phenomena in spin chains through sparse alternating fields
}

\author{
M. Cerezo, ${ }^{1}$ R. Rossignoli, ${ }^{1,2}$ N. Canosa,${ }^{1}$ and C. A. Lamas ${ }^{1}$ \\ ${ }^{1}$ Instituto de Física de La Plata, CONICET, and Departamento de Física, \\ Universidad Nacional de La Plata, C.C. 67, La Plata 1900, Argentina \\ ${ }^{2}$ Comisión de Investigaciones Científicas de la Provincia de Buenos Aires (CIC), La Plata 1900, Argentina
}

\begin{abstract}
We analyze the phase diagram of the exact ground state (GS) of spin- $s$ chains with ferromagnetic $X X Z$ couplings under $n$-alternating field configurations, i.e, sparse alternating fields having nodes at $n-1$ contiguous sites. It is shown that such systems can exhibit a non-trivial magnetic behavior, which can differ significantly from that of the standard $(n=1)$ alternating case and enable mechanisms for controlling their magnetic and entanglement properties. The boundary in field space of the fully aligned phase can be determined analytically $\forall n$, and shows that it becomes reachable only above a threshold value of the coupling anisotropy $J_{z} / J$, which depends on $n$ but is independent of the system size. Below this value the maximum attainable magnetization becomes much smaller. We then show that the GS can exhibit significant magnetization plateaus, persistent for large systems, at which the magnetization per site $m$ obeys the quantization rule $2 n(s-m)=$ integer, consistent with the Oshikawa, Yamanaka and Affleck (OYA) criterion. We also identify the emergence of field induced spin polymerization, which explains the presence of such plateaus. Entanglement and field induced frustration effects are also analyzed.
\end{abstract}

\section{INTRODUCTION}

One of the distinct hallmarks of cooperative behavior in interacting many-body quantum systems are the critical properties and phase transitions that arise when some control parameter is varied [15. In the last decades entanglement theory has unveiled new properties of these transitions, providing a deep understanding [6 13. In this scenario, the emergence of notable phenomena such as frustration 14 17 and magnetization plateaus 18 23], is typically associated with antiferromagnetic systems with competing interactions 24, 25] and non-trivial geometries 26]. However, much less is known of the critical properties that could be induced even in simple systems through general non-uniform magnetic fields or couplings. Most investigations on nonuniform fields were focused so far on the alternating or "staggered" case 27 35. Nonetheless, recent studies with more general nonuniform fields [35, 36] have shown that interesting and significant phenomena can emerge, particularly with sparse field configurations 36.

Interacting spin systems provide an adequate framework for studying such non trivial phenomena. Moreover, the possibility of simulating spin systems with tunable couplings and fields is becoming increasingly feasible due to the recent remarkable advances in quantum control technologies 37 39. In particular, the paradigmatic $X X Z$ model 1, 40 49 can emerge as effective Hamiltonian in different systems 5062 . For instance, it can be achieved in terms of superconducting charge qubits (SCQ) coupled with a SQUID (superconducting quantum interference device) 50 52. In SCQ setups, the local field parameters can be controlled by means of a gate voltage applied to each SCQ box and an external magnetic flux is used to modulate the Josephson coupling energy 50]. Other examples comprise trapped ions 38, 53, 55, cold atoms in optical lattices [37, 56, 58, photon coupled microcavities [59, quantum dots [60], etc. The $X X Z$ model has also been employed for implement- ing quantum information protocols 37 39, 60, 63, 64.

Here we will show that the application of sparse periodic alternating fields in a ferromagnetic $X X Z$ system of arbitrary spin results in novel ground state (GS) phase diagrams, which display non-trivial magnetization plateaus and entanglement properties. In the first place, the boundary in field space of the fully aligned phase, which determines the onset of GS entanglement, can be determined analytically and implies a threshold value of the coupling anisotropy, below which the maximum attainable magnetization becomes much smaller. Such boundary is independent of the system size. It is then shown that such sparse fields can induce other non-trivial magnetization plateaus, persistent for large sizes, as verified through DMRG 65 67 calculations. These plateaus are shown to satisfy the well known OYA criterion 23], which can be here explained simply through field induced polymers with definite magnetization. We also analyze other aspects like field induced frustration, single-spin magnetization and pairwise entanglement, whose results support the polymerization based picture.

The model and the $n$-alternating field configuration are described in sec. III with the boundary of the fully aligned phase and the conditions under which it can be reached discussed in IA GS magnetization diagrams are then discussed in ЩB while pairwise entanglement in एC The appendices contain the derivation of analytic expressions for the previous boundary and for entanglement measures at the boundary, and the exact analytic solution of the limit case of an XX chain under the present field configurations. Conclusion are drawn in [II]

\section{SPARSE ALTERNATING FIELD CONFIGURATIONS}

We consider a cyclic chain of $N$ spins $s$ interacting through first-neighbor $X X Z$ couplings in a non-uniform 
magnetic field along the $z$ axis. The Hamiltonian reads

$$
H=-\sum_{i=1}^{N}\left[h^{i} S_{i}^{z}+J\left(S_{i}^{x} S_{i+1}^{x}+S_{i}^{y} S_{i+1}^{y}\right)+J_{z} S_{i}^{z} S_{i+1}^{z}\right],
$$

where $h^{i}, S_{i}^{\mu}$ are the field and spin components at site $i$ (with $N+1 \equiv 1$ ) and $J, J_{z}$ the coupling strengths. As $\left[H, S^{z}\right]=0$, with $S^{z}=\sum_{j} S_{j}^{z}$ the total spin along the $z$ axis, its eigenstates can be characterized by the total magnetization $M=S_{z}(-N s \leq M \leq N s)$. We will set $J>0$, as the spectrum and entanglement properties of $H$ are the same for $\pm J$ 68]. They are also identical for $\left(\left\{h^{j}\right\}, M\right)$ and $\left(\left\{-h^{j}\right\},-M\right) 69$. It is as well convenient to use the scaled coupling strengths

$$
j_{z}=2 s J_{z}, \quad j=2 s J,
$$

as critical fields and couplings will depend just on $j_{z}$ and $j$ for different values of $s$ (see below).

We will here examine the $n$-alternating field configuration, depicted in Fig. 团 defined by

$$
h^{i}= \begin{cases}h_{1}, & i=1,2 n+1,4 n+1, \ldots \\ h_{2}, & i=n+1,3 n+1,5 n+1, \ldots, \\ 0, & \text { otherwise }\end{cases}
$$

which generalizes the standard alternating (A) case $\left(h_{1}, h_{2}, h_{1}, h_{2}, \ldots\right)$, recovered for $n=1$. For $n=2$ we obtain the "next-alternating" (NA) case $\left(h_{1}, 0, h_{2}, 0, \ldots\right)$, while for $n=3$ the "next-next-alternating" (NNA) case $\left(h_{1}, 0,0, h_{2}, 0,0, \ldots\right)$. We set in what follows $N=2 n K$, with $K$ the number of cells with $2 n$ spins.

A motivation for studying the field configurations (3) in the present system is that for $j_{z}>j>0$, they all exhibit, for any spin $s \geq 1 / 2$, a multicritical point in the GS at fields of opposite sign given by 36]

$$
h_{1}=-h_{2}= \pm h_{s}, \quad h_{s}=\sqrt{j_{z}^{2}-j^{2}},
$$

where all GS magnetizations plateaus merge: At this point the GS becomes $2 N s+1$ degenerate, with the GS's for each magnetization $M$ having all the same energy. This point generalizes the Pokrovsky-Talapov (PT)-type transition of a spin- $1 / 2$ chain in an alternating field [27. Furthermore, at this point there is a whole family of completely separable factorized (i.e. product) exact GS's [36], and the field (4) is then denoted as factorizing (or separability) field 36. It is independent of the chain size $K$ and the distance $n$ between spins with field, depending just on the scaled couplings (2). Here we will show that the field configurations (3) exhibit other interesting properties in the present system for $j_{z}<j$, being capable of inducing a non-trivial magnetic response.

\section{A. Border of the aligned phase}

A first basic question which arises for $n \geq 2$ is if such sparse fields are sufficient to induce a completely

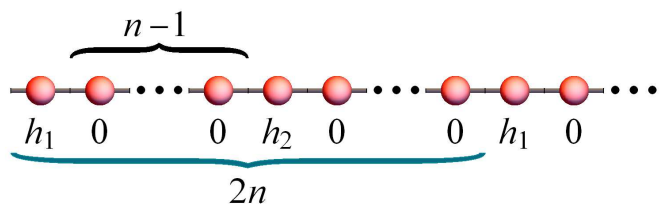

FIG. 1. Schematic representation of a spin chain with an $n$-alternating field configuration (Eq. (3)). The number of intermediate sites with no field is $n-1$. The period is $2 n$.

aligned (and hence completely separable) GS with maximum magnetization $|M|=N s$. In the standard staggered case $n=1$, such phase will always arise for sufficiently strong fields $h_{1}, h_{2}$ of the same sign, for any value of $j$ or $j_{z}$, but for $n \geq 2$ the presence of spins with zero field implies that it will not be attainable without the aid of a finite positive value of $j_{z}$, as shown below.

We will prove in fact that for $n \geq 2$, the aligned phase with $|M|=N s$ is attainable only for

$$
j_{z}>j_{z}^{c}(n)=j \cos (\pi / n), \quad n \geq 2,
$$

in which case the GS will have $M=N s$ if the fields $\left(h_{1}, h_{2}\right)$ satisfy $h_{1}+h_{2}>0$ and

$$
\left(h_{1}+\beta_{n}\right)\left(h_{2}+\beta_{n}\right)>\alpha_{n}^{2},
$$

with $h_{i}>-\beta_{n}$ such that $\left(h_{1}, h_{2}\right)$ lies above the upper branch of the hyperbola, and $M=-N s$ if $h_{1}+h_{2}<0$ and

$$
\left(h_{1}-\beta_{n}\right)\left(h_{2}-\beta_{n}\right)>\alpha_{n}^{2},
$$

with $h_{i}<\beta_{n}$ such that $\left(h_{1}, h_{2}\right)$ lies below the lower branch of a reflected hyperbola. The coefficients $\alpha_{n}, \beta_{n}$ are independent of the size $K$ and are given by

$$
\begin{aligned}
& \alpha_{n}=j \frac{\sinh \gamma}{\sinh n \gamma}=2 h_{s} \frac{j^{n}}{\left(j_{z}+h_{s}\right)^{n}-\left(j_{z}-h_{s}\right)^{n}}, \\
& \beta_{n}=j \frac{\sinh \gamma}{\tanh n \gamma}=h_{s} \frac{\left(j_{z}+h_{s}\right)^{n}+\left(j_{z}-h_{s}\right)^{n}}{\left(j_{z}+h_{s}\right)^{n}-\left(j_{z}-h_{s}\right)^{n}},
\end{aligned}
$$

where $\cosh \gamma=j_{z} / j=\Delta$ is the anisotropy and $h_{s}=$ $j \sinh \gamma$ the separability field (4), satisfying $\beta_{n}^{2}=\alpha_{n}^{2}+h_{s}^{2}$. Eqs. (8)-(9) hold and are real for both $j_{z}>j$, where $\gamma$ and $h_{s}$ are real, and also $j_{z}^{c}(n)<j_{z}<j$, where $\gamma$ and $h_{s}$ become imaginary: $\gamma=\imath \phi$, with $j_{z} / j=\cos \phi$, $h_{s}=\imath j \sin \phi$ and

$$
\alpha_{n}=j \frac{\sin \phi}{\sin n \phi}, \quad \beta_{n}=j \frac{\sin \phi}{\tan n \phi}, \quad\left(j_{z}<j\right) .
$$

In the isotropic limit $j_{z} \rightarrow j, \phi \rightarrow 0$ and $\alpha_{n}=\beta_{n} \rightarrow j / n$.

Proof. The boundary in field space $\left(h_{1}, h_{2}\right)$ of the fully aligned phase can be obtained by determining the fields at which the GS undergoes the magnetization transition $|M|=N s \rightarrow N s-1$, i.e., where the fully aligned state starts to become unstable against single spin excitations. 
The fully aligned states $|M= \pm N s\rangle$ are trivial eigenstates of $H \forall n$ in (3), with energies

$$
E_{ \pm N s}=-K s\left[ \pm\left(h_{1}+h_{2}\right)+n j_{z}\right],
$$

which are independent of $j$ and degenerate for $h_{1}+h_{2}=$ 0 . They will be the GS for sufficiently large $j_{z}$ and/or strong positive $(M=N s)$ or negative $(M=-N s)$ fields.

On the other hand, the $|M=N s-1\rangle$ eigenstate of lowest energy can be obtained by diagonalizing $H$ in the invariant subspace spanned by the $2 n W$-like [70] states with one spin down (here $S_{i}^{-}=S_{i}^{x}-i S_{i}^{y}$ ),

$$
\left|W_{i}\right\rangle=\frac{1}{\sqrt{2 s K}} \sum_{l=0}^{K-1} S_{i+2 n l}^{-}|N s\rangle, \quad i=1, \ldots, 2 n,
$$

where all sites with the same position $i$ in the cell have the same weight. These states lead to close and sizeindependent matrix elements of $\Delta H=H-E_{N s}$ :

$$
\Delta H\left|W_{i}\right\rangle=\left(j_{z}+h^{i}\right)\left|W_{i}\right\rangle-\eta_{n} j\left(\left|W_{i+1}\right\rangle+\left|W_{i-1}\right\rangle\right)
$$

where $h^{i}=\delta_{i 1} h_{1}+\delta_{i, n+1} h_{2}, \eta_{n}=1(1 / 2)$ for $n=1$ $(\geq 2)$ and $\left|W_{0}\right\rangle=\left|W_{2 n}\right\rangle,\left|W_{2 n+1}\right\rangle=\left|W_{1}\right\rangle$. A stable $M=$ $N s$ GS requires $\Delta H$ positive definite, entailing positive eigenvalues (excitation energies) of the $2 n \times 2 n$ matrix $\Delta H_{n}$ of elements

$$
\left(\Delta H_{n}\right)_{i j}=\left\langle W_{i}|\Delta H| W_{j}\right\rangle=\delta_{i j}\left(j_{z}+h^{i}\right)-\eta_{n} j \delta_{i, j \pm 1} .
$$

This implies the necessary condition

$$
\operatorname{Det}\left[\Delta H_{n}\right]>0 \text {. }
$$

Assuming $\Delta H$ positive definite for strong positive fields, the $M=N s \rightarrow N s-1$ transition then occurs at fields $\left(h_{1}, h_{2}\right)$ which are the first root of $\operatorname{Det}\left[\Delta H_{n}\right]=0$ when approached from the strong positive field limit. From Eq. (14) it is seen that this determinant has the form

$$
\operatorname{Det}\left[\begin{array}{rl}
{\left[\Delta H_{n}\right]} & =a_{n} h_{1} h_{2}+b_{n}\left(h_{1}+h_{2}\right)+c_{n} \\
& =a_{n}\left[\left(h_{1}+\beta_{n}\right)\left(h_{2}+\beta_{n}\right)-\alpha_{n}^{2}\right],
\end{array}\right.
$$

with $\beta_{n}=b_{n} / a_{n}, \alpha_{n}^{2}=\beta_{n}^{2}-c_{n} / a_{n}$ and $a_{n}, b_{n}, c_{n}$ fieldindependent. Their expressions (8)-(9) are derived in Appendix A, where it is shown that $a_{n} \geq 0$ (Eq. A2) ). Then, positivity of $\Delta H$ implies fields $\left(h_{1}, h_{2}\right)$ satisfying (6), with $h_{i}>-\beta_{n}$. And stability with respect to the $M=-N s$ GS requires $h_{1}+h_{2}>0$ (Eq. (11)). A similar procedure shows that an aligned GS with $M=-N s$ requires fields satisfying (7) with $h_{i}<\beta_{n}$ and $h_{1}+h_{2}<0$.

As $j_{z} / j$ decreases below 1 , the denominators in (10) become smaller, vanishing for $\phi \rightarrow \pi / n$ if $n \geq 2$, i.e., for $j_{z}$ approaching the critical value (5). This implies the divergence of $\alpha_{n}$ and $\beta_{n}$, and hence of the critical fields, in this limit (note that $\beta_{n}<0$ for $j_{z}^{c}(n)<j_{z}<j_{z}^{c}(2 n)$ ). The fully aligned phase becomes then unreachable for $j_{z} \leq j_{z}^{c}(n)(\phi \geq \pi / n)$. This result can also be directly derived from (14): As shown in Appendix A, the lowest eigenvalue $\lambda_{0}(n)$ of the matrix $\Delta H_{n}$ satisfies

$$
\lambda_{0}(n)<j_{z}-j \cos (\pi / n),
$$

with the upper bound reached for $h_{1}, h_{2} \rightarrow+\infty$. Thus, for $j_{z} \leq j \cos (\pi / n), \Delta H_{n}$ has a negative eigenvalue at all finite fields and the aligned state cannot be a GS. It is also verified that at the critical value $j_{z}=j \cos (\pi / n)$, $a_{n}, b_{n}$ and $c_{n}$ in Eq. (16) vanish, i.e., Det $\left[\Delta H_{n}\right]=0 \forall$ $\left(h_{1}, h_{2}\right)$ (see Appendix A).

The hyperbolas which delimit the aligned phase in Eqs. (6) - 17) also represent the onset of GS entanglement, and correspond to an entanglement transition: The $\mid M=$ $N s-1\rangle$ GS will be of the form $\sum_{i=1}^{2 n} w_{i}\left|W_{i}\right\rangle$, with $w_{i}>0$ and $\sum_{i=1}^{2 n} w_{i}^{2}=1$, which is an entangled state.

Due to the form (12) of the states $\left|W_{i}\right\rangle$, pairwise entanglement will reach full range in this sector, since the ensuing reduced state of two spins, $\rho_{i j}$, will depend just on their positions $i, j$ within the cell but not on their distance, i.e., on the number of cells between them. Since $\rho_{i j}$ is a mixed state, its entanglement can be measured through the entanglement of formation $E_{f}\left(\rho_{i j}\right)$ 71], defined as the convex roof extension of the pure state entanglement entropy. Moreover, as the present $\rho_{i j}$ can be considered as an effective two-qubit state, $E_{f}\left(\rho_{i j}\right)$ can be determined analytically by means of the concurrence 72. $C_{i i}=C\left(\rho_{i j}\right)$, which is itself an entanglement measure 73, with $C_{i j}=1$ (0) for a maximally entangled (separable) mixed state (see Appendix B for details and precise definitions of these quantities). As $\rho_{i j}$ is independent of the distance between the spins, so is the pairwise concurrence, which is given by (see again Appendix B)

$$
C_{i j}=2\left|w_{i} w_{j}\right| / K \text {. }
$$

This value saturates the monogamy relations 74,75 .

On the other hand, at the mean field level the separable fully aligned states are the trivial symmetry preserving mean field solutions, and the hyperbolas in Eqs. (6) - (7) represent the onset of the symmetry-breaking mean field phase, i.e. of degenerate mean field solutions with $\left\langle S_{i}^{\mu}\right\rangle \neq$ 0 for $\mu=x$ or $y$ (see also Appendix A). For $j_{z} \leq j_{z}^{c}(n)$ the aligned solutions are unstable at all fields.

We finally remark that for $j_{z}<j_{z}^{c}(n)$, the instability of the aligned state also holds at the single cell level, entailing that a whole interval of magnetizations (at least $N s-K+1 \leq M \leq N s$ ) also cease to be stable, as will be verified in the next section.

The first three cases. Let us now examine the particular cases $n=1,2$ and 3 in Eq. (3). In the standard staggered case $n=1$, Eqs. (8) -10) lead to

$$
\alpha_{1}=j, \quad \beta_{1}=j_{z},
$$

being then verified from (6) - (7) that the aligned phase is reachable $\forall j, j_{z}$ for sufficiently strong $h_{1}, h_{2}$. However, in the NA case $n=2$, they imply

$$
\alpha_{2}=\frac{j^{2}}{2 j_{z}}, \quad \beta_{2}=j_{z}-\frac{j^{2}}{2 j_{z}}, \quad j_{z}>0,
$$

which diverge for $j_{z} \rightarrow j_{z}^{c}(2)=0$. Increasingly stronger fields are here required to reach the aligned phase as $j_{z}$ 

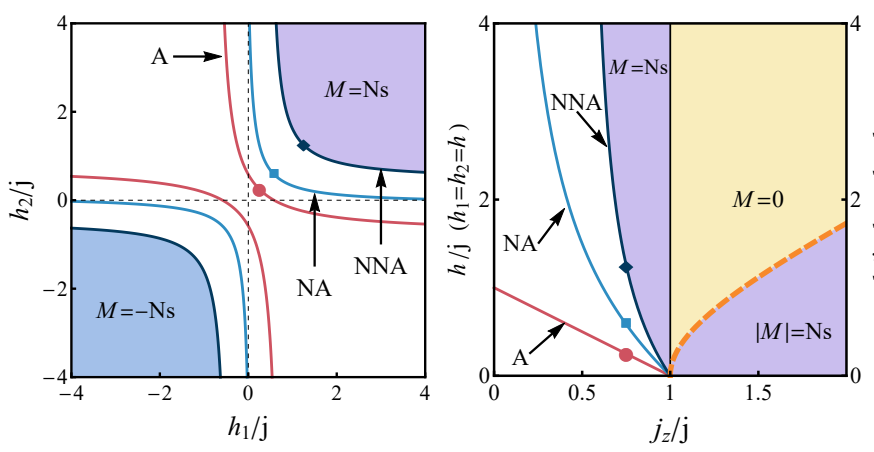

FIG. 2. GS phase diagrams for spin- $s X X Z$ chains in the alternating $(n=1, \mathrm{~A})$, "next-alternating" $(n=2, \mathrm{NA})$ and "next-next alternating" ( $n=3$, NNA) field configurations. Left: The hyperbola branches of Eqs. (6)-(7) delimiting the fully aligned $M= \pm N s$ phases at $j_{z} / j=0.75$. Colored regions indicate GS magnetization $|M|=N s$ for all three cases. Right: The threshold (23) (solid lines) of the GS aligned phase for parallel fields $h_{1}=h_{2}=h$ and $j_{z}<j$, which diverges for $j_{z} \rightarrow 0(j / 2)$ in the NA (NNA) case, and its upper limit (4) (dashed line) for antiparallel fields $h_{1}=-h_{2}=h$ and $j_{z}>j$, common for all $n$. Points indicate the thresholds for $h_{1}=h_{2}$ at $j_{z} / j=0.75$.

decreases, diverging in the $X X$ limit $j_{z}=0$. For $j_{z} \leq 0$ it becomes unreachable (see also Appendix C).

And in the NNA case $n=3$, Eqs. (8)-(10) lead to

$$
\alpha_{3}=\frac{j^{3}}{4 j_{z}^{2}-j^{2}}, \quad \beta_{3}=j_{z} \frac{4 j_{z}^{2}-3 j^{2}}{4 j_{z}^{2}-j^{2}}, \quad j_{z}>j / 2
$$

which diverge already for $j_{z} \rightarrow j_{z}^{c}(3)=j / 2$. The aligned GS cannot be reached for $j_{z} \leq j / 2$. The critical fields and couplings of these three cases are depicted in Fig. 2 with the GS magnetization diagrams shown in Fig. 3

The parallel critical field. Eqs. (6)-10) also entail that if $j_{z}^{c}(n)<j_{z}<j$, full alignment requires application of non-zero fields. For $h_{1}=h_{2}=h$, they imply

$$
|h|>h_{c}^{\|}(n)=\alpha_{n}-\beta_{n}=j \sin \phi \tan \frac{n \phi}{2},
$$

which defines a parallel critical field $h_{c}^{\|}(n)$. And if $h_{2}=$ 0 , a single field $\left|h_{1}\right|>-h_{s}^{2} / \beta_{n}=h_{c}^{\|}(2 n)$ is sufficient provided $\beta_{n}>0$, i.e. $\phi<\frac{\pi}{2 n}$, which is equivalent to $j_{z}>j_{z}^{c}(2 n)\left(h_{2}=0\right.$ in the $n$-alternating configuration is equivalent to $h_{2}=h_{1}$ in the $2 n$-alternating case).

In contrast, for $j_{z}>j$ the GS is fully aligned already at zero field $\forall n$ and lower magnetizations $|M|$ arise only for fields of opposite sign beyond the factorizing points $h_{1}=-h_{2}= \pm h_{s}$ [36], where all magnetization plateaus coalesce, as seen in the bottom panels of Fig. B The upper and lower branches of the hyperbolas (6) - (7) intersect precisely at these points $\forall n$, providing the border of the aligned phase just beyond these points. Between them, the aligned phases touch at the line $h_{1}+h_{2}=0$. Note also that for $j_{z}>j$ and $n>1, \alpha_{n}$ becomes rapidly small for large $n\left(\alpha_{n} \approx 2 h_{s}\left(\frac{j}{j_{z}+h_{s}}\right)^{n}\right)$ or large $\frac{j_{z}}{j}\left(\alpha_{n} \approx j\left(\frac{j}{2 j_{z}}\right)^{n-1}\right)$,
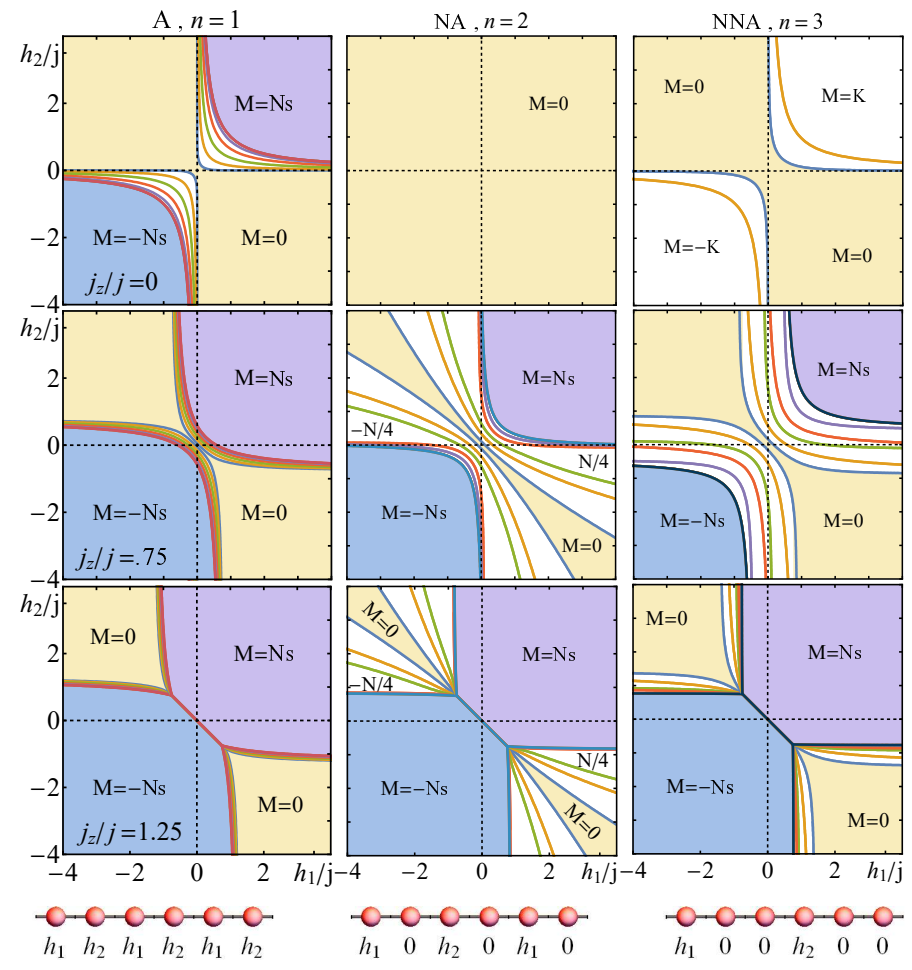

FIG. 3. GS magnetization diagram in the $\left(h_{1}, h_{2}\right)$ field plane for an $N=12$ spin-1/2 $X X Z$ chain in an $n=1$ (left), $n=2$ (center) and $n=3$ (right) field configuration (3), for anisotropies $j_{z} / j=0$ (top), 0.75 (center) and 1.25 (bottom). Curves separate different magnetizations. For $j_{z}=0$ the GS reaches all magnetizations $-N / 2 \leq M \leq N / 2$ in the A case, but remains at $M=0 \forall\left(h_{1}, h_{2}\right)$ in NA, and reaches just the $M= \pm K= \pm 2$ plateaus in NNA fields. For $j_{z} / j=0.75$, the three configurations reach all magnetizations, as now $j_{z}>j_{z}^{c}(n)$, although the NA case stands out for its wide $|M| \leq N / 4$ sectors. For $j_{z}>j$, magnetizations $|M|<N / 2$ are reached only for fields of opposite sign beyond the factorization points $h_{1}=-h_{2}= \pm h_{s}$, independent of $n$. Bottom row: schematic representation of field configurations.

implying $\beta_{n} \approx h_{s}$ and hence non alignment $(|M|<N s)$ just for $\left|h_{i}\right| \gtrsim h_{s}$ for $i=1,2$ (and $h_{1} h_{2}<0$ ).

When formally extended to all values of $j_{z}$, the antiparallel (4) and parallel (23) critical fields fully determine $\alpha_{n}$ and $\beta_{n}$, and hence the whole border of the aligned phase:

$$
\underset{\beta_{n}}{\alpha_{n}}=\frac{1}{2}\left[ \pm h_{c}^{\|}(n)-h_{s}^{2} / h_{c}^{\|}(n)\right]
$$

where $h_{c}^{\|}(n)=-j \sinh \gamma \tanh \frac{n \gamma}{2}$ for $j_{z}>j$. For $j_{z} \rightarrow j$ both vanish but $-h_{s}^{2} / 2 h_{c}^{\|}(n) \rightarrow j / n$.

\section{B. Magnetization}

A second fundamental question which arises is if magnetization plateaus with $|M|<N$ s of significant width do also emerge. For large systems the GS will indeed possess 
such plateaus (Fig. 4), at which the scaled magnetization $m=M /(N s)$ obeys the quantization rule

$$
2 n s(1-m)=q,
$$

with $q$ integer. This result can be readily understood by considering the situation where one of the fields $\left(h_{1}\right)$ is sufficiently strong so that the spin chain can be viewed approximately as $K$ polymerized subsystems consisting of $2 n-1$ spins- $s$ with a field $h_{2}$ at the central site (Fig. 1), separated by fully aligned spins. When $h_{2}$ is varied the polymer GS magnetizations $M_{2 n-1}$ will be $(2 n-1) s-q$ with $q$ integer, starting from $q=0$ when $j_{z}>j_{z}^{c}(n)$. Therefore, the total GS magnetization will be $K\{s+[(2 n-1) s-q]\}$, entailing then (25) and meaning that the plateaus in $m$ reflect essentially the polymer magnetizations. Due to the periodicity, Eq. 25) is consistent with the OYA criterion 23] (normally used in antiferromagnetic chains in uniform fields). Intermediate magnetizations arise then in the transition regions between these plateaus and imply no definite magnetization at the single cell level.

In Fig. 3 we show representative results for the GS magnetization in a small spin $1 / 2$ chain. In the standard alternating case $n=1$ (left panels), the GS reaches all magnetizations for any anisotropy $j_{z} / j$, with the fully aligned $|M|=N / 2$ sectors separated from the $M=0$ plateau by a narrow band containing all intermediate magnetizations. In contrast, in an $n=2 \mathrm{NA}$ configuration (center), it is first verified that for $j_{z}=j_{z}^{c}(2)=0$, the GS cannot be fully aligned. Moreover, it has strictly $M=0$ for all fields, as can be rigorously shown through its Jordan-Wigner fermionization [2] (see Appendix C). And for $j_{z}>0$ this configuration exhibits a noticeable behavior, showing wide $0 \leq|M| \leq N / 4$ sectors in addition to the aligned phases, with the $|M|=N / 4$ plateau persisting for large $N$ (see below). Finally, in the NNA $n=3$ case (right), it is again verified that if $j_{z} \leq j_{z}^{c}(3)=j / 2$, the GS cannot be fully aligned (top panel), reaching instead a maximum magnetization $|M|=2 s K=N / 6$ for $j_{z}=0$ (and also $0<j_{z}<j / 2$ if $s=1 / 2$ ): For strong parallel fields, spins with field become aligned while those without form essentially entangled dimers with zero magnetization, entailing $|M|=2 s K$. And when $j_{z}>j / 2$, the magnetization diagram becomes similar to that of the $n=1$ case, although with a much wider transition sector between the $M=0$ and $|M|=N / 2$ plateaus.

Previous results imply that the threshold $j_{z}^{c}(n)$ of the aligned phase is actually a critical point below which a whole interval of magnetizations cease to be reachable. This can be understood again from the strong field limit $h_{1}, h_{2} \rightarrow \infty$, where spins with field are fully aligned while those without form essentially isolated chains of $n-1$ spins, with effective fields $s J_{z}$ at the endpoints: For $n=2$ and $j_{z} \rightarrow 0^{+}$, all magnetizations $M \geq 0$ (and not just $N / 2$ and $N / 2-1$ ) of the whole chain become degenerate at strong fields, since the $M_{1}= \pm 1 / 2$ states of each of the $2 K$ single spins without field become degenerate, remaining just $M=0$ for $j_{z} \leq 0$. Similarly, for $n=3$ and

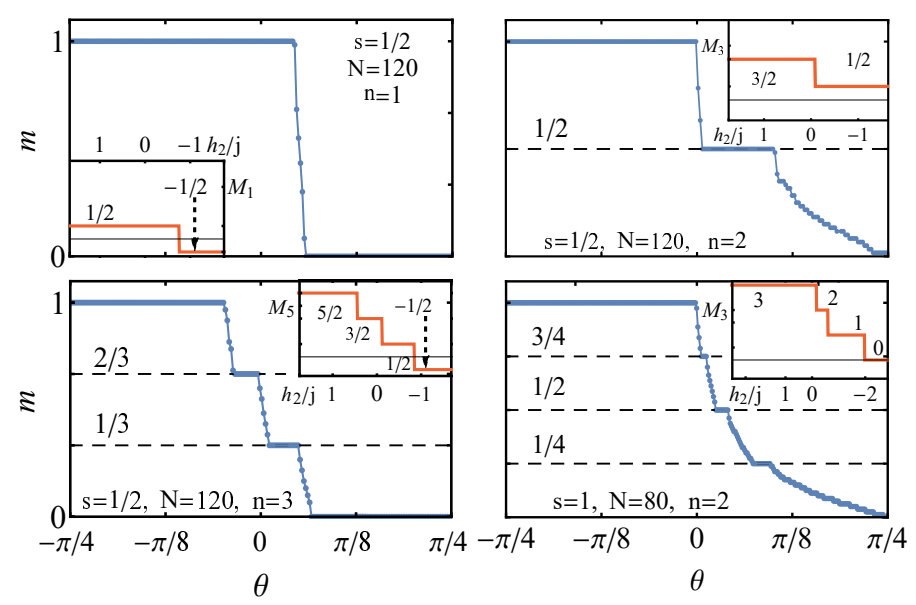

FIG. 4. Scaled GS magnetization $m=M /(N s)$ for $j_{z} / j=$ 0.75 and fields $\left(h_{1}, h_{2}\right)=4 j(\cos \theta,-\sin \theta)$. Results for $s=$ $1 / 2, N=120$ spins and $n=1,2,3$, and for $s=1, N=$ 80 spins and $n=2$, are depicted. Insets show the polymer magnetizations $M_{2 n-1}$.

$j_{z} \rightarrow j / 2$, all chain magnetizations $M \geq 2 s K=N / 6$ become degenerate at strong fields, since each pair without field may have magnetizations $M_{2}=1$ or 0 , degenerate precisely at $j_{z}=j / 2$.

In Fig. 4 we show the GS scaled magnetization for a chain of $N=120$ spins- $1 / 2$, obtained with density matrix renormalization (DMRG) 65 67, 76. In the $n=1$ case the transition region from $M=0 \rightarrow N / 2$ is again quite narrow (top left), in agreement with 25), since here the "polymer" formed for large $h_{1}$ consists of just one spin$s$, whose lower state may have only two magnetizations: $M_{1}=s$ and $-s$ (see inset), i.e. $q=0$ and $q=2 s$, leading just to $|m|=1,0$ plateaus. For $n=2$ and $j_{z}>0$, the GS possesses plateaus at $|m|=1,1 / 2$ (top right), reflecting the magnetizations $M_{3}=3 / 2,1 / 2(q=0,1)$ of the trimer formed by the three spins trapped between two aligned spins. Moreover, the trimer cannot reach $M_{3}=-1 / 2$ (except for large $h_{2} \approx-h_{1}$ ) entailing no wide $m=0$ plateau. For $n=3$, however, pentamer magnetization $M_{5}$ does reach $-1 / 2$, entailing a large $m=0$ plateau, in addition to the aligned phase $|m|=1\left(M_{5}=5 / 2, q=\right.$ 0 ) and smaller intermediate plateaus at $|m|=2 / 3,1 / 3$ $\left(M_{5}=3 / 2,1 / 2, q=1,2\right.$, bottom left). Such persistent plateaus also occur for higher spins, as seen for $s=1$ and $n=2$ (bottom right), where $|m|=1,3 / 4,1 / 2,1 / 4$, following the trimer magnetizations $M_{3}=3,2,1,0$.

Fig. 5 shows the single spin magnetization $\left\langle S_{i}^{z}\right\rangle$ of the first four spins in the chains of Fig. 3. For $s=1 / 2$, $1 / 2-\left|\left\langle S_{i}^{z}\right\rangle\right|$ is also a measure of the entanglement of spin $i$ with the rest of the chain (i.e., of the mixedness of the single spin reduced state [7] ), with $\left|\left\langle S_{i}^{z}\right\rangle\right|=0(1 / 2)$ implying maximum (zero) $i$-rest entanglement.

The spins with field will align with the field direction as $h_{i}$ increases, leading for $n=1$ to type-a (b) spin configurations for strong parallel (antiparallel) fields. However, those without field $(n \geq 2)$ exhibit a more complex 

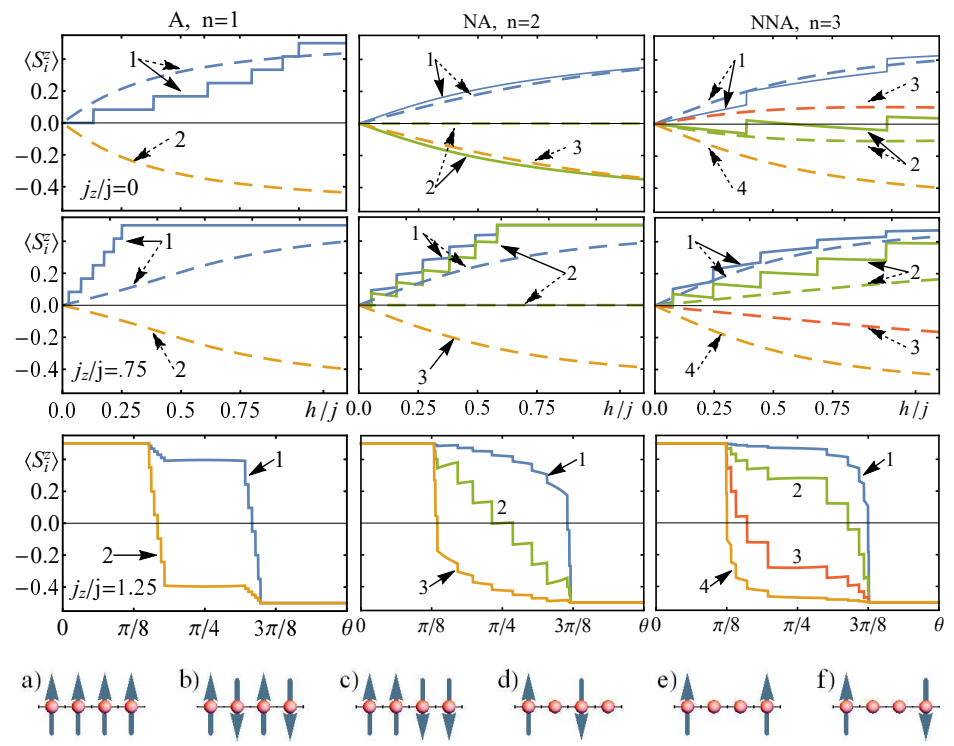

FIG. 5. Top: Individual magnetizations $\left\langle S_{i}^{z}\right\rangle$ of the first four spins in the same chains of Fig. 3 In the first and second row solid lines depict magnetizations for parallel fields $h_{1}=h_{2}=h$, and dashed lines for antiparallel fields $h_{1}=-h_{2}=h$, while in the third row fields are selected as $\left(h_{1}, h_{2}\right)=2 j(\cos \theta,-\sin \theta)$, with $\theta \in(0, \pi / 2)$. The steps in $\left\langle S_{i}^{z}\right\rangle$ reflect those of the total magnetization $M$. Bottom: schematic representation of spin configurations.

behavior. For $n=2$ and $j_{z}=0$, the total GS magnetization $M$ vanishes $\forall h_{1}, h_{2}$, implying that these spins become antialigned for $h_{1}=h_{2}$, leading to a type-b Néel configuration, but have zero magnetization $\left(\left\langle S_{i}^{z}\right\rangle=0\right)$ for $h_{1}=-h_{2}$, entailing a type-d configuration. This configuration also holds for $j_{z}>0$ if $h_{1}=-h_{2}$ (and $\left|h_{i}\right|>h_{s}$ if $j_{z}>j$ ), since $M$ still vanishes, implying that these spins become frustrated, as the attractive $S_{i}^{z} S_{i+1}^{z}$ coupling cannot be satisfied with both adjacent spins. This is a clear example of field-induced frustration, and entails maximum $i$-rest entanglement, mostly saturated with neighboring zero field spins. On the other hand, for large $h_{1}=h_{2}$ and $j_{z}>0$, they become aligned (type-a).

In contrast, for $n=3$ the two contiguous spins without field tend to form an entangled dimer, leading for $j_{z}=0$ to a type-e configuration $\left(\left\langle S_{i}^{z}\right\rangle \approx 0\right.$ for $\left.i=2,3\right)$ if $h_{1}=h_{2}$ and a type-f configuration if $h_{1}=-h_{2}$, here slightly polarized towards $\mathrm{b}$. In this case there is actually a spin configuration transition when $0<j_{z}<j_{z}^{c}(3)=j / 2$, where $\left\langle S_{i}^{z}\right\rangle$ changes sign at the central spins and the polarization evolves from type-b to type-c, crossing exactly typef. For $j_{z}>j / 2$, these central spins remain significantly entangled for antiparallel fields, polarized towards type-c, while for parallel fields they become increasingly aligned as $\left|h_{i}\right|$ and hence $|M|$ increases. Previous behaviors can also be seen at the bottom panels for $j_{z}>j$, which depict the "evolution" of $\left\langle S_{i}^{z}\right\rangle$ with $\theta=\tan ^{-1}\left(-h_{2} / h_{1}\right)$ between the fully aligned phases.

\section{Pairwise Entanglement}
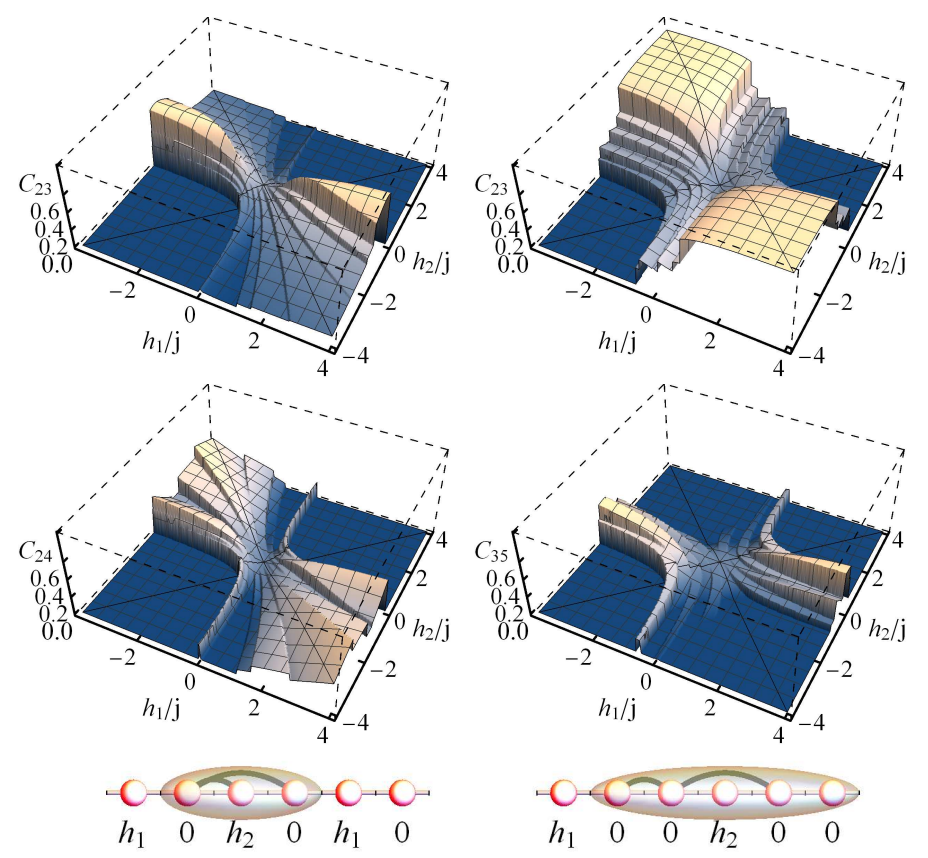

FIG. 6. Concurrence $C_{i j}$ between spins $i$ and $j$ (joined by a line in the bottom row) in the $\left(h_{1}, h_{2}\right)$ field space for the exact GS of an $N=12, s=1 / 2$ chain with $j_{z} / j=0.75$, in NA (left) and NNA (right) field configurations. The steps reflect the different total chain magnetizations. The onset of entanglement is determined by the border of the aligned phase. Bottom: Schematic representation of approximate trimerization occurring in the $\pm N / 4$ plateaus for $n=2$, and pentamerization in the $\pm N / 3$ and $\pm N / 6$ plateaus for $n=3$.

We show in Fig. [ illustrative results for the pairwise entanglement measured through the concurrence 72], in the chains of Fig. 3 for $j_{z} / j=0.75$. It is first verified that in the $n=3 \mathrm{NNA}$ case, the two contiguous spins with zero field $\left(C_{23}\right.$, top right) are highly entangled in the $M=0$ plateau, since the spins form there essentially a type-f dimerized configuration (see bottom row of Fig. 5 ). Accordingly, the concurrence $C_{35}$ of a non-contiguous pair with zero field spins (bottom right) vanishes in this plateau. In contrast, the latter becomes significant in the $|M|=4$ and $|M|=2$ plateaus $(|m|=2 / 3,1 / 3)$, where the intermediate field $h_{2}$ is weak, in agreement with the pentamerization argument.

On the other hand, in the $n=2 \mathrm{NA}$ case, $C_{23}$ (spin without field and spin with field $h_{2}$, top left) is clearly significant in the $|M|=N / 4$ plateaus emerging for small $\left|h_{2}\right|$, and small or zero in the same plateaus emerging for small $\left|h_{1}\right|$ and strong $\left|h_{2}\right|$, supporting the trimerization argument. This is verified in $C_{24}$ (bottom left), which is also significant (zero) when $C_{23}$ is large (small) in these plateaus, entailing essentially no entanglement between trimers. $C_{24}$ is also non-negligible at the $M=0$ plateau, where nearest spins with no field become entangled due 
to the field induced frustration. It is also confirmed that all concurrences are finite at the $|M|=N s-1$ band, in agreement with Eq. (19).

\section{CONCLUSIONS}

We have shown that $n$-alternating field configurations can lead to novel GS phase diagrams which differ significantly from those of the standard alternating case. They can exhibit non-trivial magnetization plateaus associated with field induced frustration and polymerization phenomena, which persist for large sizes as verified by DMRG calculations. These plateaus satisfy a quantization rule compatible with the OYA criterion and are shown to stem from field induced polymers with definite magnetization, where spins trapped between spins with fields become highly entangled among themselves but are essentially disentangled with spins in another polymer. Exact analytic expressions for the boundary in field space of the fully aligned phase, valid for all $n$, were also derived, and imply a critical $n$-dependent anisotropy $j_{z}^{c}(n) / j$ below which the aligned phase together with a whole interval of GS magnetizations become unreachable even for arbitrarily strong fields. The boundary of the aligned phase represents in addition the onset of GS entanglement (as well that of the symmetry-breaking phase at the mean field level), with pairwise entanglement acquiring there full range. These results open new possibilities for applications of finite chains with simple interactions under controllable fields, such as entanglement tuning and plateaus formation at rational values of the scaled magnetization, and pave the way to study the emergence of critical phenomena induced through non-uniform fields within more general architectures and couplings.

\section{ACKNOWLEDGMENTS}

We thank Dr. J. M. Matera for useful discussions. The authors acknowledge support from CONICET (MC, NC, CAL) and CIC (RR) of Argentina.

\section{Appendix A: Border of the aligned phase in the $n$-alternating spin- $s X X Z$ system}

We first prove Eqs. (8)- (9). In the standard alternating case $n=1, \Delta H_{n}$ in 115 is just a $2 \times 2$ matrix,

$$
\Delta H_{1}=\left(\begin{array}{cc}
h_{1}+j_{z} & -j \\
-j & h_{2}+j_{z}
\end{array}\right),
$$

and a trivial calculation yields $a_{1}=1, b_{1}=j_{z}$ and $c_{1}=$ $j_{z}^{2}-j^{2}$ in (16), with $\alpha_{1}=j, \beta_{1}=j_{z}$ (Eq. (20)). In this case the lowest eigenvalue of $\Delta H_{n}$ is just $\lambda_{0}(1)=$ $j_{z}+\frac{h_{1}+h_{2}}{2}-\sqrt{\left(\frac{h_{1}-h_{2}}{2}\right)^{2}+j^{2}}$, and Eq. (6) can be directly obtained from the condition $\lambda_{0}(1)>0$.
For general $n \geq 2$, evaluation of $\operatorname{Det}\left[\Delta H_{n}\right]$ in Eq. (16) yields

$$
a_{n}=\left(d_{n-1}\right)^{2}, \quad b_{n}=d_{2 n-1},
$$

and $c_{n}=d_{2 n}-\frac{j^{2}}{4} d_{2 n-2}-2 \frac{j^{2 n}}{4^{n}}$, where

$$
d_{n}=\left|\begin{array}{ccccc}
j_{z} & -j / 2 & 0 & 0 & \ldots \\
-j / 2 & j_{z} & -j / 2 & 0 & \ldots \\
& & \ddots & & \\
& & & \ddots & \\
0 & \ldots & 0 & -j / 2 & j_{z}
\end{array}\right|
$$

is the determinant of an $n \times n$ Toeplitz 78] tridiagonal matrix $M_{n}$ of elements $j_{z} \delta_{i j}-\frac{j}{2} \delta_{i, j \pm 1}$. It then satisfies

$$
d_{n+1}=j_{z} d_{n}-(j / 2)^{2} d_{n-1},
$$

for $n \geq 1$, with $d_{1}=j_{z}, d_{0} \equiv 1$, i.e., $\left(\begin{array}{c}d_{n+1} \\ d_{n}\end{array}\right)=A^{n}\left(\begin{array}{l}j_{z} \\ 1\end{array}\right)$, with $A=\left(\begin{array}{cc}j_{z}-j^{2} / 4 \\ 1 & 0\end{array}\right)$. Hence, for any $n \geq 1$, diagonalization of $A$, which has eigenvalues $\frac{1}{2}\left(j_{z} \pm h_{s}\right)=\frac{1}{2} j e^{ \pm \gamma}$, with $h_{s}=\sqrt{j_{z}^{2}-j^{2}}=j \sinh \gamma$ and $\cosh \gamma=j_{z} / j$, leads to

$$
d_{n}=\frac{\left(j_{z}+h_{s}\right)^{n+1}-\left(j_{z}-h_{s}\right)^{n+1}}{2^{n+1} h_{s}}=\frac{j^{n}}{2^{n}} \frac{\sinh [(n+1) \gamma]}{\sinh \gamma} .
$$

Eqs. A2 - A5 then lead to $a_{n}=\left(\frac{j}{2}\right)^{2 n-2} \frac{\sinh ^{2} n \gamma}{\sinh ^{2} \gamma}, b_{n}=$ $\left(\frac{j}{2}\right)^{2 n-1} \frac{\sinh 2 n \gamma}{\sinh \gamma}$ and $c_{n}=4\left(\frac{j}{2}\right)^{2 n} \sinh ^{2} n \gamma$, implying $\alpha_{n}=$ $2\left(\frac{j}{2}\right)^{n} / d_{n-1}$, i.e. Eq. (8), with $\beta_{n}=b_{n} / a_{n}$ given by (9).

Now, it is apparent from (16) and previous expressions that the matrix $\Delta H_{n}$ is positive definite for $j_{z}>j$ and positive fields $h_{1}, h_{2}\left(a_{n}>0, b_{n}>0, c_{n}>0 \forall\right.$ real $\left.\gamma\right)$. On the other hand, at the threshold value (5), $\gamma=\imath \pi / n$ and Eqs. A2 - A5 lead to $a_{n}=b_{n}=c_{n}=0$, i.e. $\operatorname{Det}\left[\Delta H_{n}\right]=0 \forall h_{1}, h_{2}$, indicating the presence of a vanishing eigenvalue of $\Delta H_{n}$ and hence the loss of stability of the aligned $M=N s$ GS.

The eigenvalues of $\Delta H_{n}$ represent of course excitation energies constructed from single spin excitations when $\Delta H_{n}$ is positive definite. The eigenvalue equation $\operatorname{Det}\left[\Delta H_{n}-\lambda \mathbb{1}\right]=0$ can be explicitly obtained from Eq. (16) and the previous expressions for $a_{n}, b_{n}, c_{n}$, replacing $j_{z} \rightarrow j_{z}-\lambda$ and $\gamma \rightarrow \imath \phi:$ It reads

$$
\frac{\sin ^{2} n \phi}{\sin ^{2} \phi}\left[h_{1} h_{2}+\frac{j\left(h_{1}+h_{2}\right) \sin \phi}{\tan n \phi}-j^{2} \sin ^{2} \phi\right]=0,
$$

where $\cos \phi=\left(j_{z}-\lambda\right) / j$. It is first seen that A6 is fulfilled for $\phi=\pi k / n, k=1, \ldots, n-1$, implying the $n-1$ field-independent eigenvalues

$$
\lambda_{k}(n)=j_{z}-j \cos (\pi k / n), \quad k=1, \ldots, n-1 .
$$

The lowest one, $\lambda_{1}(n)$, vanishes precisely at the threshold (5), becoming negative for $j_{z}<j_{z}^{c}(n)=j \cos (\pi / n)$. In 
addition, the bracket in A6 leads to the remaining $n+1$ field-dependent eigenvalues. The lowest one is obtained for $\phi=\phi_{0}<\pi / n$ (and $\phi_{0}>0$ ), leading to

$$
\lambda_{0}(n)=j_{z}-j \cos \phi_{0}<\lambda_{1}(n)=j_{z}-j \cos \pi / n,
$$

with equality approached only at strong fields $h_{1}, h_{2} \gg j$ (where $\phi_{0} \approx \pi / n-\frac{h_{1}+h_{2}}{n h_{1} h_{2}} \sin (\pi / n)$, approaching $\pi / n$ for $\left.h_{1}, h_{2} \rightarrow+\infty\right)$. Thus, for $j_{z}>j_{z}^{c}(n), \Delta H_{n}$ is always positive definite at sufficiently strong fields $\left(\lambda_{0}(n)>0\right)$, while for $j_{z} \leq j_{z}^{c}(n)$, it is non-positive $\left(\lambda_{0}(n)<0\right)$ at all finite fields and the aligned state can no longer be a GS.

Replacing $j_{z} \rightarrow j_{z}-\lambda$ in A5 , it is also seen that the eigenvalues of the $(n-1) \times(n-1)$ Toeplitz matriz $M_{n-1}$ are just those of Eq. A7 78. This matrix is just the block of $(\Delta H)_{n}$ associated with the $n-1$ contiguous spins with no field, which become decoupled from the aligned spins with field for $h_{1}, h_{2} \rightarrow \infty$. Hence, $-j \cos \pi / n$ represents the lowest energy of the $n-1$ spins trapped between the two aligned spins at $j_{z}=0$ and magnetization $(n-1) s-1$,

While a positive definite matrix $\Delta H_{n}$ is in principle a necessary condition for stability of the $M=N s$ GS, it turns out to be sufficient for $h_{1}+h_{2}>0$, since in this case the GS magnetization decreases in steps of length 1 from its maximum $M=N s$ as the fields $h_{1}, h_{2}$ decrease from $+\infty$ (Fig. 3). The only exception occurs for $j_{z}>j$ along the line $h_{1}+h_{2}=0$ between the factorizing fields (see bottom panels in Fig. 3), where the aligned states $M= \pm N s$ become degenerate GS's if $\left|h_{i}\right|<h_{s}$, and all GS magnetizations plateaus merge if $\left|h_{i}\right|=h_{s}$.

Finally, we note that in the mean field approximation, the onset of the symmetry-breaking phase is again determined by the fields where the matrix $\Delta H_{n}$ ceases to be positive definite, since it is constructed from single spin excitations. A symmetry-breaking product state $\left|\Psi_{\mathrm{mf}}\right\rangle \propto$ $e^{-\imath \sum_{i} \theta_{i} S_{i}^{y}}|M=N s\rangle$ becomes in fact $\approx|N s\rangle+\sum_{i} w_{i}\left|W_{i}\right\rangle$ for small $\theta_{i}$, with $w_{i}=\theta_{i} \sqrt{s K / 2}$. Hence, a non-positive $\left\langle\psi_{\mathrm{mf}}|\Delta H| \psi_{\mathrm{mf}}\right\rangle$ is then equivalent to $\Delta H_{n}$ not being positive definite.

\section{Appendix B: Reduced states and entanglement in} the $M=N s-1$ GS

The $|M=N s-1\rangle$ GS will have the form

$$
|N s-1\rangle=\sum_{i=1}^{2 n} w_{i}\left|W_{i}\right\rangle
$$

where $\left|W_{i}\right\rangle$ are the states (12) and the coefficients $w_{i}$ are obtained from the diagonalization of the matrix $\Delta H_{n}$ of elements (14) $\left(\sum_{i}\left|w_{i}\right|^{2}=1\right.$, with $w_{i}>0 \forall i$ for $J>$ 0 ). From the form (12) of the states $\left|W_{i}\right\rangle$, it becomes apparent that the reduced state $\rho_{k l}=\operatorname{Tr}_{\overline{k l}}|N s-1\rangle\langle N s-1|$ of any two distinct spins $k \neq l$ in the state (B1) will depend just on their positions $i, j$ within the cell each spin belongs, but not on their absolute distance $|k-l|$. Since the reduced state will also commute with the total spin $S_{k l}^{z}=S_{k}^{z}+S_{l}^{z}$ of the pair, it will be given, for $M=N s-1$, by ( $K=N / 2 n$ is the number of cells)

$$
\rho_{i j}=\left(\begin{array}{cccc}
1-\frac{\left|w_{i}\right|^{2}+\left|w_{j}\right|^{2}}{K} & 0 & 0 & 0 \\
0 & \frac{\left|w_{j}\right|^{2}}{K} & \frac{w_{j} w_{i}^{*}}{K} & 0 \\
0 & \frac{w_{i} w_{j}^{*}}{K} & \frac{\left|w_{i}\right|^{2}}{K} & 0 \\
0 & 0 & 0 & 0
\end{array}\right),
$$

in the subspace spanned by the states $\{|s s\rangle,|s, s-1\rangle, \mid s-$ $1, s\rangle,|s-1, s-1\rangle\}$, where $|s-1\rangle=\frac{1}{\sqrt{2 s}} S^{-}|s\rangle$. Eq. B2. is valid for any $s$ and $i, j=1, \ldots, 2 n$. It can then be always considered as a mixed state of an effective two-qubit system, as just states $|s\rangle$ and $|s-1\rangle$ are involved at each spin. A similar expression holds for the reduced state in the $M=-N s+1$ GS in the corresponding subspace.

The state B2 is a mixed state with two non-zero eigenvalues $p_{i j}=\left(\left|w_{i}\right|^{2}+\left|w_{j}\right|^{2}\right) / K$ and $1-p_{i j}$. Its entropy $S\left(\rho_{i j}\right)=-\operatorname{Tr} \rho_{i j} \log _{2} \rho_{i j}$ is the entanglement entropy of the pair with the rest of the chain. On the other hand, the entanglement between both spins can be measured through its entanglement of formation [7], defined as the convex roof extension of the pure state entanglement entropy: For a general mixed state $\rho \equiv \rho_{A B}$, it is the minimum of the average entanglement over all decompositions of $\rho$ as convex mixture of pure states:

$$
E_{f}(\rho)=\operatorname{Min}_{\left\{q_{\alpha},\left|\Psi_{\alpha}\right\rangle\right\}} \sum_{\alpha} q_{\alpha} E\left(\left|\Psi_{\alpha}\right\rangle\right),
$$

where $\sum_{\alpha} q_{\alpha}\left|\Psi_{\alpha}\right\rangle\left\langle\Psi_{\alpha}\right|=\rho, q_{\alpha} \geq 0, \sum_{\alpha} q_{\alpha}=1$, and $E\left(\left|\Psi_{\alpha}\right\rangle\right)=S\left(\rho_{A}^{\alpha}\right)=S\left(\rho_{B}^{\alpha}\right)$ is the entanglement entropy of $\left|\Psi_{\alpha}\right\rangle\left(\rho_{A(B)}^{\alpha}=\operatorname{Tr}_{B(A)}\left|\Psi_{\alpha}\right\rangle\left\langle\Psi_{\alpha}\right|\right.$ are the reduced states $)$.

While the evaluation of Eq. (B3) in the general case is a computationally hard problem, for a two-qubit mixed state $\rho$ it can be analytically determined through the concurrence $C(\rho)$ 72], defined as in Eq. [B3] with $E\left(\left|\Psi_{\alpha}\right\rangle\right) \rightarrow C\left(\left|\Psi_{\alpha}\right\rangle\right)=\sqrt{S_{2}\left(\rho_{A}^{\alpha}\right)}=\sqrt{S_{2}\left(\rho_{B}^{\alpha}\right)}$, where $S_{2}(\rho)=2\left(1-\operatorname{Tr} \rho^{2}\right)$ is the linear entropy. For a twoqubit state $\rho$ the concurrence can be calculated as 72

$$
C(\rho)=\operatorname{Max}\left[2 \lambda_{\max }-\operatorname{Tr} R, 0\right], \quad R=\left[\rho^{1 / 2} \tilde{\rho} \rho^{1 / 2}\right]^{1 / 2},
$$

where $\lambda_{\max }$ denotes the largest eigenvalue of $R$ and $\tilde{\rho}=$ $\sigma_{y} \otimes \sigma_{y} \rho^{*} \sigma_{y} \otimes \sigma_{y}$ is the spin flipped density, with $\sigma_{y}$ the Pauli matrix. Eq. B3 then becomes 72

$$
E_{f}(\rho)=-\sum_{\nu= \pm} q_{\nu} \log _{2} q_{\nu}, \quad q_{ \pm}=\frac{1 \pm \sqrt{1-C^{2}(\rho)}}{2}
$$

and is just an increasing convex function of $C(\rho)$, with $E_{f}(\rho)=C(\rho)=1(0)$ for a maximally entangled (separable) two-qubit state. For a pure state $\rho=|\Psi\rangle\langle\Psi|$, $C(\rho)=\sqrt{S_{2}\left(\rho_{A(B)}\right)}$ and $E_{f}(\rho)$ becomes the standard entanglement entropy $S\left(\rho_{A(B)}\right)$. The concurrence is itself a proper entanglement monotone 73. and satisfies a monogamy inequality 74,75 . 
In the case of the state (B2), the pair concurrence $C_{i j}=C\left(\rho_{i j}\right)$ obtained from Eq. (B4) becomes just $C_{i j}=2\left|\left(\rho_{i j}\right)_{23}\right|=2\left|w_{i} w_{j}\right| / K$ and is then given by Eq. (19). These concurrences saturate the monogamy inequality, namely

$$
\sum_{l \neq i} C_{i l}^{2}=4 \frac{\left|w_{i}\right|^{2}}{K}\left(1-\frac{\left|w_{i}\right|^{2}}{K}\right)=C_{i, \text { rest }}^{2},
$$

where $C_{i, \text { rest }}^{2}=S_{2}\left(\rho_{i}\right)=2\left(1-\operatorname{Tr} \rho_{i}^{2}\right)$ is the tangle of single spin $i$ with the rest of the chain, with

$$
\rho_{i}=\left(\begin{array}{cc}
1-\left|w_{i}\right|^{2} / K & 0 \\
0 & \left|w_{i}\right|^{2} / K
\end{array}\right),
$$

the reduced state of spin $i$ in the state B22. For a general state we have instead $\sum_{l \neq i} C_{i l}^{2} \leq C_{i, \text { rest }}^{2}$ 74, 75].

While Eq. (19) is valid for any spin $s$ due to the form (B2) of the reduced pair state, in general states the pairwise entanglement of formation for spin $s \geq 1$ will not be analytically computable. Instead, we can use as computable quantifier the negativity $N(\rho)$ 79], defined as the absolute value of the sum of the negative eigenvalues of the partial transpose of $\rho \equiv \rho_{A B}$. According to the Peres criterion 80, $N(\rho)>0$ implies entanglement (though the converse does not hold in general). In the $|M|=N s-1$ region, the negativity $N_{i j}=N\left(\rho_{i j}\right)$ determined by the state B2 is, setting $\gamma_{i j}=1-\left(\left|w_{i}\right|^{2}+\left|w_{j}\right|^{2}\right) / K$,

$$
N_{i j}=\frac{1}{2}\left(\sqrt{\gamma_{i j}^{2}+4\left|w_{i}\right|^{2}\left|w_{j}\right|^{2} / K^{2}}-\gamma_{i j}\right),
$$

with $N_{i j} \rightarrow C_{i j}^{2} / 2$ for large $K$.

Due to the symmetry $w_{n+1+i}=w_{n+1-i}$ valid for $i=$ $1, \ldots, n-1$ in the exact GS under cyclic conditions, the coefficients $w_{i}$ in (B1) can be obtained by diagonalizing an effective $(n+1) \times(n+1)$ matrix $\Delta H_{n}^{\prime}$. Altogether there are just $(n+1)$ distinct coefficients $w_{i}$ and hence just $(n+1)(n+2) / 2$ distinct pairwise concurrences and negativities for general $h_{1}, h_{2}$ in the $|M|=N s-1$ GS.

\section{Appendix C: Exact solution of the $X X$ chain in $n$-alternating field configurations}

When $J_{z}=0$, the $X X Z$ model reduces to the $X X$ model. For $s=1 / 2$, the ensuing Hamiltonian can be mapped exactly to a bilinear fermionic form in the annihilation $c_{j}^{\dagger}$ and creation $c_{j}$ operators by means of the Jordan-Wigner transformation [2] $c_{j}^{\dagger}=$ $S_{j}^{+} \exp \left(-\imath \pi \sum_{k=1}^{j-1} S_{k}^{+} S_{k}^{-}\right)$for each value of the fermionic number parity (i.e., the $S_{z}$-parity)

$$
P \equiv \exp (\imath \pi \mathbf{N})=\sigma= \pm 1,
$$

where $\mathbf{N}=\sum_{j=1}^{N} c_{j}^{\dagger} c_{j}=S^{z}+N / 2$ is the fermion number operator. This leads to

$$
H=-\sum_{j}\left[h_{j}\left(c_{j}^{\dagger} c_{j}-1 / 2\right)-\eta_{j}^{\sigma} \frac{J}{2}\left(c_{j+1}^{\dagger} c_{j}+c_{j}^{\dagger} c_{j+1}\right)\right]
$$

where, for cyclic conditions, $\eta_{j}^{-}=1 \forall j$ and $\eta_{j}^{+}=1(-1)$ for $j \leq N-1(j=N)$. After a discrete Fourier transform of the fermion operators, it can be expressed as a sum of $K 2 n \times 2 n$ matrices $\mathbf{H}_{k}$ :

$$
\begin{aligned}
H & =-\sum_{k=1-\delta_{\sigma 1} / 2}^{K-\delta_{\sigma 1} / 2} \mathbf{c}_{k}^{\prime \dagger} \cdot \mathbf{H}_{k} \mathbf{c}_{k}^{\prime}-\epsilon \\
\mathbf{H}_{k} & =\left(\begin{array}{ccc}
h^{+}+J \cos \omega_{k} & h^{-} & \ldots \\
h^{-} & h^{+}+J \cos \left(\omega_{k}+\frac{\pi}{n}\right) & \ldots \\
\vdots & \vdots & \ddots
\end{array}\right), \\
& =\mathbf{D}_{k}+\mathbf{A},
\end{aligned}
$$

with $\mathbf{c}_{k}^{\prime \dagger}=\left(c_{k}^{\prime \dagger}, c_{k+N /(2 n)}^{\prime \dagger}, \ldots, c_{k+(2 n-1) N /(2 n)}^{\prime \dagger}\right), \mathbf{D}_{k}$ a diagonal matrix of elements $\left(\mathbf{D}_{k}\right)_{i i}=J \cos \left(\omega_{k}+\right.$ $\left.\frac{\pi(i-1)}{n}\right)$, A a circulant matrix specified by the vector $\left(h^{+}, h^{-}, h^{+}, h^{-}, \ldots\right)$, and

$$
h^{ \pm}=\frac{h_{1} \pm h_{2}}{2 n}, \epsilon=\frac{N h^{+}}{2}, \omega_{k}=2 \pi k / N .
$$

Eq. C3) shows that the Fourier transformed $n$ alternating field configuration leads to off diagonal hopping terms specifying the allowed momentum values. The index $k$ is half-integer (integer) for $\sigma=1(-1)$.

Due to the parity dependence of the energy levels, the number of GS magnetization transitions is associated to the number of times the single particle energies change sign 83. Hence, field values at which single particle energies vanish can be determined by solving

$$
\operatorname{Det}\left[\mathbf{H}_{k}\right]=0 \text {, }
$$

with $k=1 / 2,1, \ldots, K$.

For standard alternating fields $n=1$, Eq. (C4) becomes

$$
\mathbf{H}_{k}=\left(\begin{array}{cc}
h^{+}+J \cos \omega_{k} & h^{-} \\
h^{-} & h^{+}-J \cos \omega_{k}
\end{array}\right),
$$

yielding the well known single particle energies 81 87

$$
{ }_{1} \lambda_{k}^{ \pm}=h^{+} \pm \sqrt{\left(h^{-}\right)^{2}+J^{2} \cos ^{2} \omega_{k}} .
$$

In this case

$$
\operatorname{Det}\left[\mathbf{H}_{k}\right]=h_{1} h_{2}-J^{2} \cos ^{2} \omega_{k},
$$

and Eq. C7) determines $N / 2$ hyperbolas in the $\left(h_{1}, h_{2}\right)$ field space, meaning that the GS will then exhibit definite magnetization plateaus ranging from $|M|=0$ to $|M|=$ $N / 2$. In particular, for $k=N / 2$ the lowest $\sigma=-1$ parity level becomes negative and we recover exactly the hyperbola $h_{1} h_{2}=j^{2}$ of the $N / 2 \rightarrow N / 2-1$ transition, in agreement with Eqs. (6)-(7) for $n=1$ and $j_{z}=0$. For $n \geq 2$ the expressions for the eigenvalues are more involved.

In the NA $n=2$ case, the determinant of $\mathbf{H}_{k}$ is

$$
\operatorname{Det}\left[\mathbf{H}_{k}\right]=\frac{J^{4}}{4} \sin ^{2}\left(2 \omega_{k}\right),
$$


which becomes zero only for $k=N / 4$ and implies at least one identically zero single particle energy. The latter means that there is no single particle energy which changes sign as the fields are varied and indicates that there should be no GS magnetization transition. Furthermore, we now prove the following lemma:

Lemma 1. The GS of a finite $X X$ spin system in a $n=2$ next-alternating field configuration is a nondegenerate half-filled state with definite magnetization $M=0$, $\forall h_{1}, h_{2}$.

Proof: We first start by comparing the number of energy levels with negative single particle energies within each parity $\sigma$ and their ensuing lowest energy $E_{\sigma}$. Since $\operatorname{Det}\left[\mathbf{H}_{k}\right] \geq 0 \forall k$ [Eq. [C11] ] then each matrix $\mathbf{H}_{k}$ is either positive (or negative) semi-definite, or it has two positive and two negative eigenvalues. However, since the determinant of any leading principal minor connecting $i$ with $i+2$ is $-J^{2} \cos ^{2}\left(\omega_{k}\right), \mathbf{H}_{k}$ cannot be positive nor negative semi-definite. In the $\sigma=1$ subspace, $\operatorname{Det}\left[\mathbf{H}_{k}\right]>0$ $\forall k=\{1 / 2, \ldots K-1 / 2\}$, entailing that there are always $N / 2=2 K$ negative single particle energies, whereas for $\sigma=-1$ there are $N / 2-1$, as one of the eigenvalues of $\mathbf{H}_{N / 4}$ is identically zero. Due to this small, albeit important, difference in the number of negative energy levels, $E_{1}<E_{-1} \forall h_{1}, h_{2}$. While this result can be numerically verified, for $h_{2}= \pm h_{1}= \pm h$ a series expansion of the energy difference between the lowest energies of each parity, $\Delta E=E_{-1}-E_{1}$, shows that $\Delta E>0 \forall h$. Likewise, for strong fields a second order perturbation treatment in the couplings shows that the $M=0$ eigenstate is the GS $\forall J$.
[1] S. Sachdev, Quantum Phase Transitions (Cambridge University Press, Cambridge, England, 1999); S. Sachdev, Nature Physics 4, 173 (2008).

[2] E. Lieb, T. Schultz, D. Mattis, Ann. Phys. 16407 (1961).

[3] M. Vojta, Rep. Prog. Phys. 66, 2069 (2003).

[4] N. Laflorencie, I. Affleck, M. Berciu, J. Stat. Mech. P12001 (2005).

[5] Z. Wang, T. Lorenz, D. I. Gorbunov, P. T. Cong, Y. Kohama, S. Niesen, O. Breunig, J. Engelmayer, A. Herman, J. Wu, K. Kindo, J. Wosnitza, S. Zherlitsyn, A. Loidl, Phys. Rev. Lett. 120, 207205 (2018).

[6] A. Osterloh, L. Amico, G. Falci, R. Fazio, Nature (London) 416, 608 (2002).

[7] T.J. Osborne, M.A. Nielsen, Phys. Rev. A 66, 032110 (2002).

[8] G. Vidal, J. I. Latorre, E. Rico, A. Kitaev, Phys. Rev. Lett. 90, 227902 (2003).

[9] A.R. Its, B-Q Jin, V.E. Korepin, J. Phys. A Math. Gen. 38, 2975 (2005).

[10] L. Amico, R. Fazio, A. Osterloh, V. Vedral, Rev. Mod. Phys. 80, 517 (2008).

[11] J. Eisert, M. Cramer, M. B. Plenio, Rev. Mod. Phys. 82, 277 (2010).

[12] J. Stasinska, B. Rogers, M. Paternostro, G. De Chiara, A. Sanpera, Phys Rev A 89, 032330 (2014).

[13] N. Blanc, J. Trinh, L. Dong, X. Bai, A. A. Aczel, M. Mourigal, L. Balents, T. Siegrist, A. P. Ramirez, Nature Physics 14, 273 (2018).

[14] C. Lacroix, P. Mendels, F. Mila, Introduction to Frustrated Magnetism: Materials, Experiments, Theory (Springer, Berlin, 2013).

[15] A. Sen(De), U. Sen, J. Dziarmaga, A. Sanpera, M. Lewenstein, Phys. Rev. Lett. 101, 187202 (2008).

[16] F. Michaud, T. Coletta, S. R. Manmana, J. D. Picon, F. Mila, Phys. Rev. B 81, 014407 (2010).

[17] S.M. Giampaolo, G. Gualdi, A. Monras, F. Illuminati, Phys. Rev. Lett. 107, 260602 (2011); U. Marzolino, S.M. Giampaolo, F. Illuminati, Phys. Rev. A 88, 020301(R) (2013).

[18] A. Honecker, J. Schulenburg, J. Richter, J. Phys.: Condens. Matter 16, S749 (2004).

[19] A. Tanaka, K. Totsuka, X. Hu, Phys. Rev. B 79, 064412
(2009).

[20] M. Takigawa, F. Mila, Magnetization plateaus, Springer Series in Solid-State Sciences, Vol. 164 (Springer, 2011), Chap. 10, pp. 241267.

[21] C. A. Lamas, S. Capponi, P. Pujol, Phys. Rev. B 84, 115125 (2011); F. Elias, M. Arlego, C. A. Lamas, Phys. Rev. B 95, 214426 (2017).

[22] H. Hu, C. Cheng, Z. Xu, H.-G. Luo, S. Chen, Phys. Rev. B 90, 035150 (2014).

[23] M. Oshikawa, M. Yamanaka, I. Affleck, Phys. Rev. Lett. 78, 1984 (1997).

[24] H. Zhang, C.A. Lamas, M. Arlego, W. Brenig, Phys. Rev. B 93, 235150 (2016).

[25] C.K. Majumdar, D.K. Ghosh, J. Math. Phys. 10, 1399 (1969).

[26] S. Nishimoto, N. Shibata, C. Hotta, Nat. Commun. 4, 2287 (2013).

[27] F.C. Alcaraz, A.L. Malvezzi, J. Phys. A 28, 1521 (1995).

[28] M.Asoudeh, V.Karimipour, Phys. Rev. A 71, 022308 (2005).

[29] G.-F. Zhang, S.-S. Li, Phys. Rev. A 72, 034302 (2005).

[30] N. Canosa, R. Rossignoli, J.M. Matera, Phys. Rev. B 81, 054415 (2010).

[31] S. Mahdavifar, J. Abouie, J. Phys. Condens. Matter 23, 246002 (2011).

[32] A.A. Zvyagin, Phys. Rev. B 97, 214425 (2018); A.A. Zvyagin, Quantum Theory of One-Dimensional Spin Systems, (Cambridge, U.K., 2010).

[33] C. Li, G. Zhang, Z. Song Phys. Rev. A 94, 052113 (2016).

[34] T. Chanda, T. Das, D. Sadhukhan, A. K. Pal, A. Sen De, U. Sen, Phys. Rev. A 94, 042310 (2016).

[35] M. Cerezo, R. Rossignoli, N. Canosa, Phys. Rev. B 92, 224422 (2015); Phys. Rev. A 94, 042335 (2016).

[36] M. Cerezo, R. Rossignoli, N. Canosa, E. Ríos, Phys. Rev. Lett. 119, 220605 (2017).

[37] M. Lewenstein, A. Sanpera, V. Ahufinger, Ultracold Atoms in Optical Lattices: Simulating Quantum ManyBody Systems (Oxford University Press, NY, 2012).

[38] I. M. Georgescu, S. Ashhab, F. Nori, Rev. Mod. Phys. 86, 153 (2014).

[39] Principles and Methods of Quantum Information Technologies, eds. Y. Yamamoto, K. Semba (Springer, NY, 
2016).

[40] C.N. Yang, C.P. Yang, Phys. Rev. 150, 321 (1966).

[41] J.D. Johnson, M. McCoy, Phys. Rev. A 6, 1613 (1972).

[42] F. C. Alcaraz, S. R. Salinas, W. F. Wreszinski, Phys. Rev. Lett. 75, 930 (1995); F. C. Alcaraz, A. Saguia, M. S. Sarandy, Phys. Rev. A 70, 032333 (2004).

[43] D.V. Dmitriev, V.Y. Krivnov, A.A. Ovchinnikov, Phys. Rev. B 65, 172409 (2002); D.V. Dmitriev, V.Y. Krivnov, A.A. Ovchinnikov, A. Langari, Zh. Eksp. Teor. Fiz. 122, 624 (2002) [JETP 95, 538 (2002)].

[44] S.-J. Gu, H.-Q. Lin, Y.-Q. Li, Phys. Rev. A 68, 042330 (2003).

[45] N. Canosa, R. Rossignoli, Phys. Rev. A 73, 022347 (2006); E. Ríos, R. Rossignoli, N. Canosa, J. Phys. B 50, 095501 (2017).

[46] R. Jafari, A. Langari, Phys. Rev. B 76, 014412 (2007).

[47] O. Breunig, M. Garst, E. Sela, B. Buldmann, P. Becker, L. Bohatý, R. Müller, T. Lorenz, Phys. Rev. Lett. 111, $187202(2013)$.

[48] J. Reisons, E. Mascarenhas, V. Savona, Phys. Rev. B 96, 165137 (2017).

[49] J.Ren, Y.Wang, W.L.You, Phys. Rev. A 97, 042318 (2018).

[50] G. Xu, G. Long, Sci. Rep. 4, 6814 (2014).

[51] Y. Salathe, M. Mondal, M. Oppliger, J. Heinsoo, P. Kurpiers, A. Potocnik, A. Mezzacapo, U. Las Heras, L. Lamata, E. Solano, S. Filipp, A. Wallraff, Phys. Rev. X 5, 021027 (2015).

[52] M. Roth et al., arXiv:1808.04666.

[53] D. Porras, J.I. Cirac, Phys. Rev. Lett. 92, 207901 (2004).

[54] R. Blatt, C.F. Roos, Nat. Phys. 8, 277 (2012).

[55] I. Arrazola, J.S. Pedernales, L. Lamata, E. Solano, Sc. Rep. 6, 30534 (2016).

[56] O.V. Marchukov, A.G. Volosniev, M. Valiente, D. Petrosyan, N.T. Zinner, Nat. Comms. 7, 13070 (2016); A.G. Volosniev, D. Petrosyan, M. Valiente, D.V. Fedorov, A.S. Jensen, N.T. Zinner, Phys. Rev. A 91, 023620 (2015).

[57] S. Whitlock, A. W. Glaetzle, P. Hannaford, J. Phys. B: At. Mol. Opt. Phys. 50, 074001 (2017).

[58] T.L. Nguyen, J.M. Raimond, C. Sayrin, R. Cortinas, T. Cantat-Moltrecht, F. Assemat, I. Dotsenko, S. Gleyzes, S. Haroche, G. Roux, T. Jolicoeur, M. Brune, Phys. Rev. X 8, 011032 (2018).

[59] C.Noh, D.G.Angelakis, Rep. Prog. Phys. 80, 016401 (2017).

[60] Y.P. Shim, S. Oh, X. Hu, M. Friesen, Phys. Rev. Lett. 106, 180503 (2011).

[61] R. Toskovic et al, Nat. Phys. 12, 656 (2016).

[62] O. Breunig, M. Garst, A. Klümper, J. Rohrkamp, M. Turbull, T. Lorenz, Sci. Adv. 3, 3773 (2017).

[63] S.C. Benjamin, S. Bose, Phys. Rev. A 70, 032314 (2004).
[64] A. Bayat, S. Bose, Phys. Rev. A 81, 012304 (2010); L. Banchi, A. Bayat, P. Verrucchi, S. Bose, Phys. Rev. Lett. 106, 140501 (2011).

[65] S. R. White, Phys. Rev. B 48, 10345 (1993).

[66] A. Kolezhuk, R. Roth, U. Schollwöck, Phys. Rev. Lett. 77, 5142 (1996); Phys. Rev. B 55, 8928 (1997).

[67] U. Schollwöck, Rev. Mod. Phys. 77, 259 (2003); Ann. Phys. 326, 96 (2011).

[68] The sign of $J$ can be changed by local rotations of angle $\pi$ around the $z$ axis at even (or odd) sites $j$.

[69] These cases are linked by a global rotation of angle $\pi$ around the $x$ or $y$ axis, which leaves the coupling unchanged.

[70] W. Dür, G. Vidal, J.I. Cirac, Phys. Rev. A 62, 062314 (2000).

[71] C.H. Bennett, D.P. DiVincenzo, J.A. Smolin, W.K. Wootters, Phys. Rev. A 54, 3824 (1996).

[72] S. Hill, W.K. Wootters, Phys. Rev. Lett. 78, 5022 (1997); W.K. Wootters, Phys. Rev. Lett. 80, 2245 (1998).

[73] G. Vidal, J. Mod. Opt. 47, 355 (2000).

[74] V. Coffman, J. Kundu, W.K. Wootters, Phys. Rev. A 61, 052306 (2000).

[75] T.J. Osborne, F. Verstraete, Phys. Rev. Lett. 96, 220503 (2006).

[76] We usually perform 20 sweeps keeping up to 900 states.

[77] In a pure state with definite total magnetization $M$ along $z$, the single spin reduced state $\rho_{i}$ commutes with $S_{i}^{z} \forall s$. For $s=1 / 2$ its eigenvalues are then $1 / 2 \pm\left\langle S_{i}^{z}\right\rangle$, and its purity $\operatorname{Tr} \rho_{i}^{2}=\frac{1}{2}+2\left\langle S_{i}^{z}\right\rangle^{2}$.

[78] A. Böttcher, S.M. Grudsky, Spectral Properties of Banded Toeplitz Matrices, SIAM (2005).

[79] G. Vidal, R.F. Werner, Phys. Rev. A 65, 032314 (2002); K. Zyczkowski, P. Horodecki, A. Sanpera, M. Lewenstein, Phys. Rev. A 58, 883 (1998).

[80] A. Peres, Phys. Rev. Lett. 77, 1413 (1996); M. Horodecki, P. Horodecki, R. Horodecki, Phys. Lett. A 223, 1 (1996).

[81] J.H. H. Perk, H.W. Capel, M.J. Zuilhof, Th.J. Siskens, Phys. A 81, 319 (1975).

[82] K. Okamoto, K.Yasumura, J. Phys. Soc. J. 59, 993 (1990).

[83] N. Canosa, R. Rossignoli, Phys. Rev. A 75, 032350 (2007).

[84] S. Deng, G. Ortiz, L. Viola, EPL 84, 67008 (2008).

[85] U. Divakaran, A. Dutta, D. Sen, Phys. Rev. B 78, 144301 (2008).

[86] A. De Pasquale, P. Facchi, Phys. Rev. A 80, 032102 (2009).

[87] A. Dutta, G. Aeppli, B.K. Chakrabarti, U. Divakaran, T.F.Rosenbaum, D. Sen, Quantum phase transitions in transverse field spin models: From statistical physics to quantum information Cambridge Univ. Press, UK (2015). 